\title{
Prognosis of railway ballast degradation for turnouts using track-side accelerations
}

\author{
Barkhordari, Pegah; Galeazzi, Roberto; Blanke, Mogens
}

Published in:

Proceedings of the Institution of Mechanical Engineers, Part O: Journal of Risk and Reliability

Link to article, DOI:

10.1177/1748006x20901410

Publication date:

2020

Document Version

Peer reviewed version

Link back to DTU Orbit

Citation (APA):

Barkhordari, P., Galeazzi, R., \& Blanke, M. (2020). Prognosis of railway ballast degradation for turnouts using track-side accelerations. Proceedings of the Institution of Mechanical Engineers, Part O: Journal of Risk and Reliability. https://doi.org/10.1177/1748006×20901410

\section{General rights}

Copyright and moral rights for the publications made accessible in the public portal are retained by the authors and/or other copyright owners and it is a condition of accessing publications that users recognise and abide by the legal requirements associated with these rights.

- Users may download and print one copy of any publication from the public portal for the purpose of private study or research.

- You may not further distribute the material or use it for any profit-making activity or commercial gain

- You may freely distribute the URL identifying the publication in the public portal 


\title{
Prognosis of railway ballast degradation for turnouts using track side accelerations
}

\author{
Pegah Barkhordari ${ }^{1}$, Roberto Galeazzi ${ }^{1}$ and Mogens Blanke ${ }^{1}$
}

\begin{abstract}
Maintenance of railway turnouts is a major cost driver for any infrastructure manager, which performs it based on preventive or periodic policies to guarantee minimum downtime of the infrastructure. Ballast maintenance activities, such as tamping, are scheduled by thresholding indicators derived from track geometry data in agreement with European standards. Current industrial practice adopts the same procedures for the evaluation of track geometry degradation in open track and turnouts. This lack of specificity may determine unnecessary maintenance actions with detrimental effects to the turnout dynamical performance. By employing track-side measurements of track acceleration a probabilistic method for turnout's ballast quality monitoring was derived in (1). The paper further develops such monitoring system by establishing a mapping between its output and the standard indicator of track geometry quality used by infrastructure managers to schedule ballast maintenance. This results in a predictor of track geometry quality with variable prediction horizon that is functional to the railway infrastructure manager for the predictive planning of ballast maintenance.
\end{abstract}

\section{Keywords}

Data-driven monitoring, prognosis, ballast degradation, track geometry, railway turnout, change detection

\section{Introduction}

Mammoth financial resources are spent annually on maintenance of railway networks to ensure safety, continuous availability and quality. Turnouts are vulnerable elements in the reliability chain and their performance is preeminent for the overall network dependability. With one-third of total track maintenance expenses spent on turnouts, these are heavy posts on maintenance budgets (2). The proper performance of a turnout as a complex system highly depends on the health state of all of its sub-components. Failure of any of these components may lead to break-down of a turnout, and in the worst case to derailing of a passing train. Recorded data in the UK in 2009 (3, Chapter 7) showed that ballast failure was among the top three failure modes of turnouts. It is hence of importance for safety, train regularity and economy to guarantee the ballast quality at all time. Periodic and preventive ballast maintenance are currently the most common strategies adopted by the infrastructure managers to ensure the necessary stiffness of the track support layer. These maintenance policies are known to be overly conservative in favoring risk reduction over track utilization. To improve the cost efficiency of railway maintenance, predictive policies should be developed with the support of novel condition indicators that could monitor actual track (ballast) degradation and reliably predict future degradation trends.

Various methods have been proposed in the past for assessing the quality of railway track. Researchers and engineers have used direct and indirect techniques, ranging from visual inspection to recently developed processing techniques and prediction models, to facilitate the decision making and enable efficient maintenance planning. The ground penetrating radar (GPR), the cone penetration test
(CPT) and visual inspection are the most common direct methods utilized to evaluate the infrastructure condition. These direct techniques have few drawbacks discussed in (4; 5; 6). Indirect techniques mainly exploit track stiffness as an indicator of the track quality and they rely on vibration data collected by measurement vehicles or trackside measurement systems to measure/estimate it. The rolling stiffness measurement vehicle (RSMV) (7), the track loading vehicle (TLV) (8) and the moving rail car (9) are commonly used measurement vehicles for assessing track stiffness. Such measurements were accompanied by GPR measurements (10) to correlate RSMV and TLV measurements with the substructure assessment. Track-side measurement systems have been recently used with the goal of monitoring the track infrastructure. Le Pen et al. (11) proposed a method for obtaining the track modulus as a factor influencing the rate of degradation. The method was based on the combination of Fourier analysis of measured data collected by sensors mounted on sleepers with a previously developed mathematical model (beam on elastic foundation). Suitability of track-side measurement systems for monitoring of turnout components was discussed in (12). Frequency analysis of acceleration data collected under the crossing nose was proposed for evaluating behavior of the track components. Barkhordari et al. (6) exploited

\footnotetext{
${ }^{1}$ Technical University of Denmark, Department of Electrical Engineering, Automation and Control Group, Building 326, Elektrovej, $2800 \mathrm{Kgs}$. Lyngby.
}

Corresponding author:

Pegah Barkhordari

Email: pebark@elektro.dtu.dk 
the train-induced vibration data collected by a trackside measurement system and developed statistical lowcomplexity behavioural models suitable for the condition monitoring of railway turnouts. The modeling scheme was based on the combination of the Empirical Mode Decomposition (EMD) with the subspace identification algorithm N4SID. Statistical models were obtained to characterize the behavior of the first and second track resonance frequencies as relevant features to monitor track components degradation.

Railway infrastructure managers monitor the quality of the track utilizing geometry parameters that are measured using the so-called track geometry (recording) car. These geometry parameters are recommended by the European standard EN 13848-6 (13) together with the operational ranges needed to assess the quality of the railway lines. The ballast quality is indirectly appraised through the computation of the standard deviation of the track longitudinal level in the wavelength range from 3 to $25 \mathrm{~m}\left(\sigma_{H}\right)$, which is used to determine the track quality class and the time for performing the ballast tamping activities $(14 ; 15)$. Track geometry measurements usually occur on a quarterly basis or more seldom in relation to track utilization. The time interval trades off between the relatively slow development time of track degradation phenomena and the cost associated with performing such measurement campaigns. In high-speed lines the measurements are performed every two-weeks $(16 ; 17)$ due to their more stringent safety requirements. However, track quality degradation is a non-uniform nonlinear phenomenon developing differently from site to site in connection with actual track quality and exogenous impacting factors as condition of the subsoil and climate. Therefore, more frequent measurements could provide additional insight into the track/ballast quality degradation process, but this will further increase the O\&M cost with current adopted methods.

Challenges emerge when the method recommended by the European standard is to be applied for the monitoring of turnouts; in particular

1. Data collected in subsequent geometry measurement campaigns need to be spatially aligned to correctly determine which track sections are suffering degradation. There is an uncertainty associated with the spatial information provided by the Global Navigation Satellite system (GNSS) onboard the track recording car (18), which is used for geotagging the data points. The position accuracy may suffice to reliably estimate $\sigma_{H}$ when calculated over the $200 \mathrm{~m}$ section length, as specified in the European standard. However, the analyses of degradation over the much shorter sections of a turnout (i.e. switch panel, closure panel and crossing panel) can be strongly affected by misalignment errors that could lead to inaccurate classification and assessment of the ballast quality.

2. The track quality analysis performed over $200 \mathrm{~m}$ long track sections cannot provide a truthful picture of the turnout condition (19) since this track length includes either more than one turnout or a turnout combined with an open track section. This in general leads to an under or overestimate of the ballast quality.
The first challenge has been overcome by means of different methods for the spatial alignment of the track geometry data in turnouts $(20 ; 21)$. Exploiting the aligned data, a number of approaches have been developed for analyzing the degradation of turnouts. Two methods were proposed in (22) for analyzing time series of the longitudinal level collected over 5 years. The methods were defined based on variations of several parameters extracted from the profile of the longitudinal level over the considered period. Suitability of the presented methods for the degradation analysis and the maintenance decision making was discussed. An analysis based on the power spectral density (PSD) of the longitudinal level measured at four different turnouts in the Swedish railway network was proposed in (23) and its outcome was compared with the Chinese PSD standard to determine the track quality condition. It was discussed that the obtained results can help the infrastructure managers plan and prioritize the tamping activities. In (24) fractal dimensioning of the longitudinal level measurements collected from fifteen turnouts was performed to define a ballast quality index, which was then used together with other endogenous and exogenous parameters to develop a universal ballast degradation model. The authors addressed the use of track vibrations as additional information to improve the accuracy of the long-term forecasting by the model.

While some of the obstacles to the reliable assessment of turnout degradation based on the measured track geometry data have been overcome in the previous studies, the proposed methods are still limited to the availability of the track geometry measurements. As mentioned earlier, both frequent and occasional executions of the measurement campaigns have their disadvantages. One possible solution to these problems is to continuously assess the track quality along turnouts exploiting data collected by a track-side measurement system. This has been carried out in (1), where a ballast quality indicator has been developed exploiting train-induced vibrations. Although the ballast quality indicator proposed in the latter study can well indicate degradation trends of the ballast layer along the turnout, its value cannot directly be linked to a specific degradation state (e.g. not degraded, partly degraded, degraded, severely degraded). Therefore, the present study focuses on the operationalization of this quality indicator to enable the determination of the ballast quality class in accordance with the classification provided in the European standard. This is achieved by establishing a mapping between the outcome of the ballast quality indicator proposed in (1) and the track quality indicator $\sigma_{H}$ adopted by the infrastructure managers.

The paper is structured as follows: first, the track-side measurement system is presented; then the monitoring architecture presented in (1) is briefly revisited; subsequently a regression model is obtained to map the proposed indicator into $\sigma_{H}$ thereby obtaining an estimator of $\sigma_{H}$; last the obtained estimator is evaluated on the available track geometry data for the turnout at hand to determine the ballast quality class. Tack geometry and vibration data collected over a period of two years at a turnout of the Danish railway infrastructure are used to perform the analysis. 


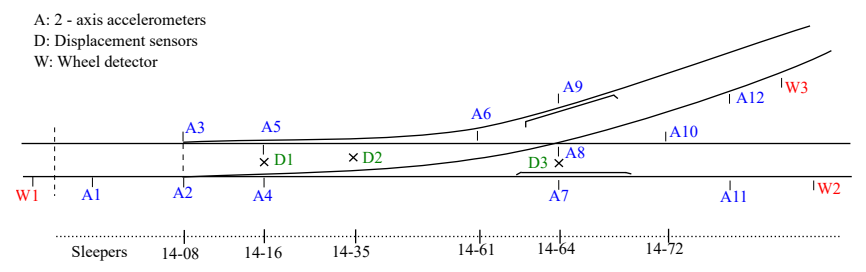

Figure 1. Layout of the sensors location along the turnout at Tommerup station (Fyn - Denmark). The data utilized in this work refer to vertical accelerations measured by accelerometers A2 (switch panel), A7 (crossing panel) and A11 (13 meters after the tip of crossing).
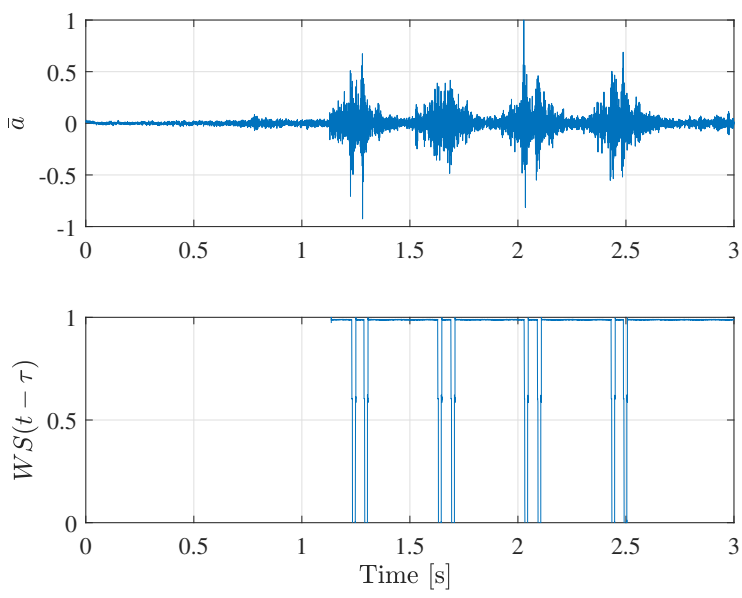

Figure 2. The track acceleration response to an IC3 passenger train and the wheel detector signal as measured at location A7 (crossing panel).

\section{Experimental set-up}

The track-side measurement set-up consists of a data acquisition system to which 3 wheel detectors, 122 axis accelerometers (measurement range: $\pm 500 \mathrm{~g}$ ) and 3 displacement sensors (measurement range: $\pm 20 \mathrm{~mm}$ ) are connected. Signals from the sensor are conditioned and temporarily stored in the data acquisition system. The schematic provided in Fig. 1 shows the location of the sensors along the instrumented turnout at Tommerup station (Fyn - Denmark). The wheel detectors are used to enable automatic data recording whenever a train passes through the turnout. The accelerometers are magnetically mounted on the rail web to measure train-induced vertical and lateral accelerations. The displacement sensors are mounted on sleepers and measure the vertical displacement. The signals are collected at the sampling rate of $F_{s}=20 \mathrm{kHz}$. Traininduced vertical acceleration data recorded at the switch panel (A2), crossing panel (A7) and exit of turnout (A11) are considered for the analysis of ballast degradation. As an example, a measurement of acceleration collected at location A7 and the time-shifted output of the wheel detector are shown in Fig. 2, where $\bar{a}=a / \max (|a|)$. The timeshifted output of the wheel detector is exploited for precisely determining the time instants at which the train bogies are passing through the measurement location. The time delay $\tau$ is calculated based on the distance between the wheel detector and the accelerometer, and the train speed. Although
Table 1. Limit values of $\sigma_{H}$ in $D_{1}$ domain (wavelength 3-25 m), (13)

\begin{tabular}{lccccc}
\hline Speed $(\mathrm{km} / \mathrm{h})$ & \multicolumn{5}{c}{ Track quality class } \\
\hline & A & B & C & D & E \\
\hline $120<V \leq 160$ & $<0.65$ & 0.85 & 1.4 & 1.85 & $>1.85$ \\
\hline
\end{tabular}

trains of different types and speeds pass through the turnout, only IC3 trains with the speed ranging from 158 to $160 \mathrm{~km} / \mathrm{h}$ are considered in the analysis because, as shown in (25), trains with lower axle loads and higher speeds better excite the track resonance frequencies.

\section{Longitudinal level measurement}

The quality assessment of the railway track can be carried out in two different ways (26): (1) analysing the track geometry; (2) evaluating the substructure condition. The former is the most common approach for track quality assessment and it relies on the determination of track geometry indicators by means of which the maintenance activities are planned. Track geometry cars are commonly used by railway infrastructure managers to measure longitudinal level, alignment, track gauge, cross level and twist as typical parameters indicating the track condition. The standard deviation $\sigma_{H}$ of the track longitudinal level averaged between right and left rails is one of the most significant geometry indicators recommended by the European standard EN13848-6 (13) for track quality assessment and ballast maintenance decision making. According to the standard, for a given train speed range the track geometry quality can be classified into five classes from A to E depending on the value of $\sigma_{H}$, where class A and class E represent the best and the worst $10 \%$ of the distribution of European track quality, respectively. Given the maximum speed allowed at Tommerup station, the values of $\sigma_{H}$ corresponding to different track quality classes are provided in Table 1.

A universal measuring vehicle (UFM 120) shown in Fig. 3 is currently used by the Danish railway infrastructure manager, Banedanmark, to measure track geometry parameters at every $25 \mathrm{~cm}$ along track sections in the railway network. Inspection of the track by using the measuring vehicle is usually performed 3-4 times a year, with an average time interval of 100 days between the measurement campaigns. The geometry parameters recorded as time-series are represented in the spatial domain utilizing the information provided by a GNSS. Due to uncertainty in the position information recorded by the GNSS, a spatial shift in the data measured over the same track section during two different measurement campaigns can be observed. Therefore, for a given location along the track, in order to be able to compare the values of parameters measured during different campaigns, a synchronization of the time-series in the spatial domain must be performed. This has been done in the present study by means of spatial alignment according to the methods described in $(24 ; 21)$.

Figure 4 shows the standard deviation of the longitudinal level calculated based on measurements collected by the universal measuring vehicle in eight measurement campaigns over the period of two years. In Fig. 4(a) 


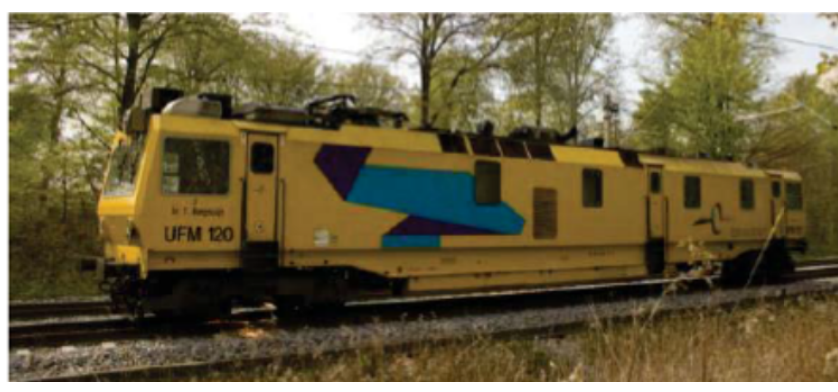

Figure 3. The UFM120 track recording car (the picture is courtesy of the Danish infrastructure manager, Banedanmark).

different sections with length of $200 \mathrm{~m}$ including the turnout under study are taken into account. It can be seen that the obtained track quality is strongly dependent on the position of the considered section. The significant difference between the first two sections and section three is due to the fact the former contain two turnouts and open track while the latter contains only one turnout and open track. This clearly manifests the raised issue of the unsuitability of using $200 \mathrm{~m}$ long sections to establish a truthful picture of the turnout track quality. Consequently, scheduling turnout ballast maintenance activities based on the $\sigma_{H}$ value as currently computed may lead to either unnecessary or delayed actions. It is hence of paramount importance to localize the analysis to the neighborhood of critical locations along the turnout. Figure 4(b) shows the value of $\sigma_{H}$ computed for locations A2, A7 and A11 using a $10 \mathrm{~m}$ long section around that location. This section length is chosen according to the sensitivity analysis carried out in (27), which indicates that a single accelerometer can reliably monitor a $10 \mathrm{~m}$ long section along the turnout. The trend of $\sigma_{H}$ clearly shows that the tamping activity has improved the quality of the track at all three locations along the turnout, although in different measure. According to the results shown in this figure and the information provided in Table 1, the track quality class has changed from class $\mathrm{B}$ to class $\mathrm{A}$ at location $\mathrm{A} 2$, and from class $\mathrm{C}$ to class $\mathrm{B}$ at locations $\mathrm{A} 7$ and $\mathrm{A} 11$. Moreover, the results obtained from the measurement campaigns subsequent to the tamping event show the gradual degradation of the ballast quality during the entire period. Figure 4(b) distinctly illustrates how track quality degradation occurs unevenly along the turnout and how, when compared with Fig. 4(a), it can be wrongly estimated if data are spurious.

\section{Implementation of the monitoring tool}

The proposed monitoring tool is designed based on a statistical model built through estimations of the first track resonance frequency $\omega_{n, 1}$, which represents the ballast quality in terms of stiffness. In order to estimate the first track resonance frequency, the method introduced in (6) is applied to the track acceleration data induced by a pool of IC3 trains. To perform the statistical analysis, data collected within a 2-year period from September 2016 to September 2018 are taken into account. Figure 5 shows the obtained histogram and probability plots for two monthly periods, a month before and a month after a tamping event, at the switch

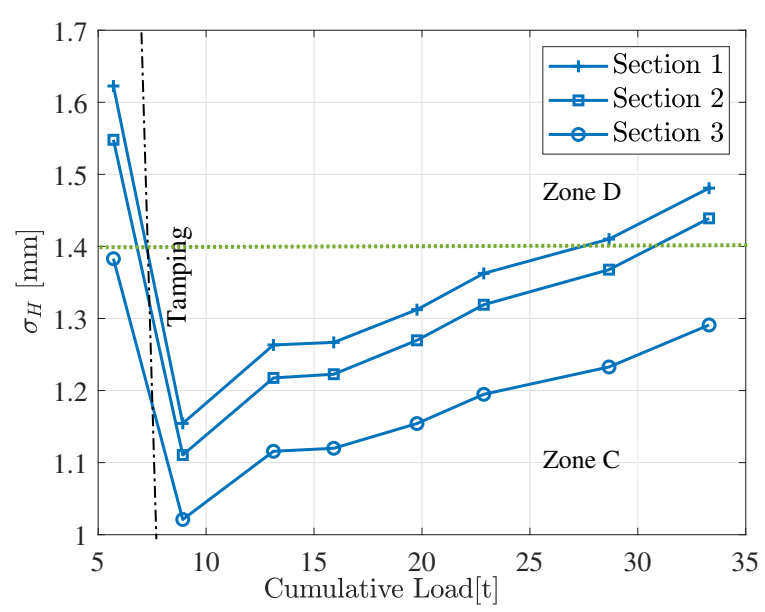

(a) Standard deviation for three $200 \mathrm{~m}$ long track sections including the turnout at Tommerup station.

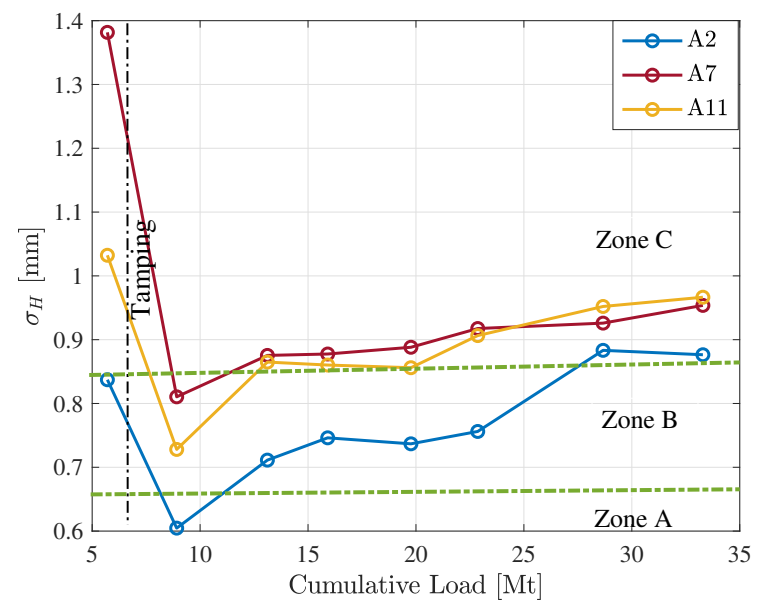

(b) Standard deviation of $10 \mathrm{~m}$ long track sections along the turnout at Tommerup station.

Figure 4. Standard deviation of the track longitudinal level computed for eight consecutive track geometry campaigns over a two-year period.

panel (location A2). The distinct change in $\omega_{n, 1}$ indicates the effect of the tamping event on the improvement of the ballast quality. In order to find a proper model describing the statistical behavior of the data, suitability of different types of distributions such as generalized extreme value (GEV), Normal and Weibull distributions have been evaluated. The goodness of fit calculated using the Kolmogorov-Smirnov test ( $\mathrm{p}$-value) is provided in Table 2, and it indicates that the GEV distribution is the best fit to the data. This is also shown in Fig. 5(b) where the GEV distribution $(\mathcal{G})$ properly approximates the behavior of the estimated first track resonance frequency before $\left(\boldsymbol{\omega}_{n 1 B T}\right)$ and after $\left(\boldsymbol{\omega}_{n 1 A T}\right)$ the tamping event.

The probability density function of $\mathcal{G}$ distribution is given by,

$\mathcal{G}(x)=\frac{1}{\sigma} \exp \left[-\left(1+\xi \frac{x-\mu}{\sigma}\right)^{-\frac{1}{\xi}}\right]\left(1+\xi \frac{x-\mu}{\sigma}\right)^{-1-\frac{1}{\xi}}$ 


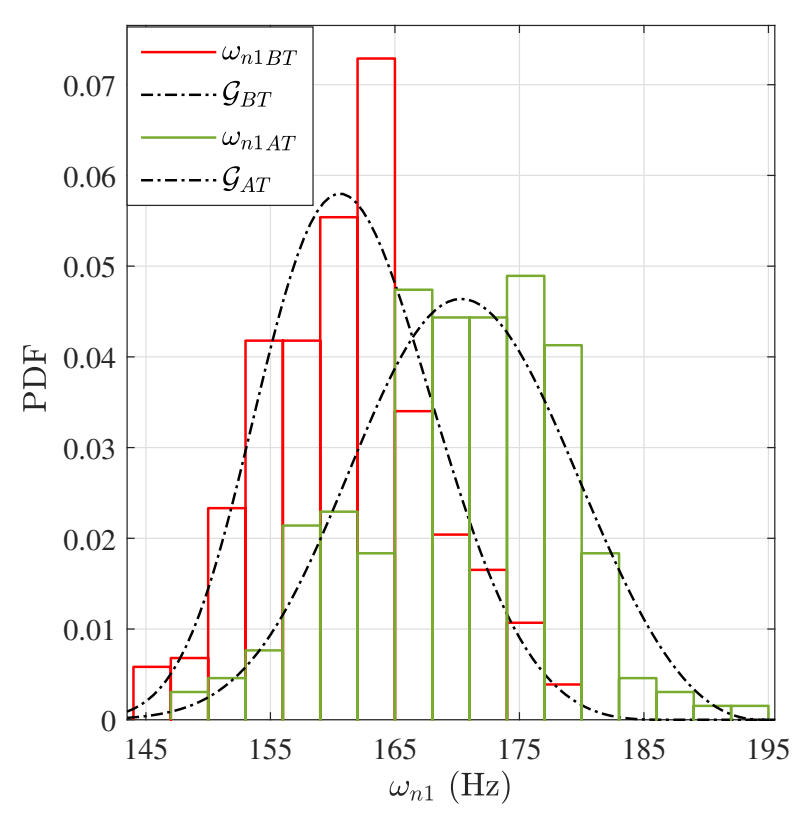

(a) Histogram.

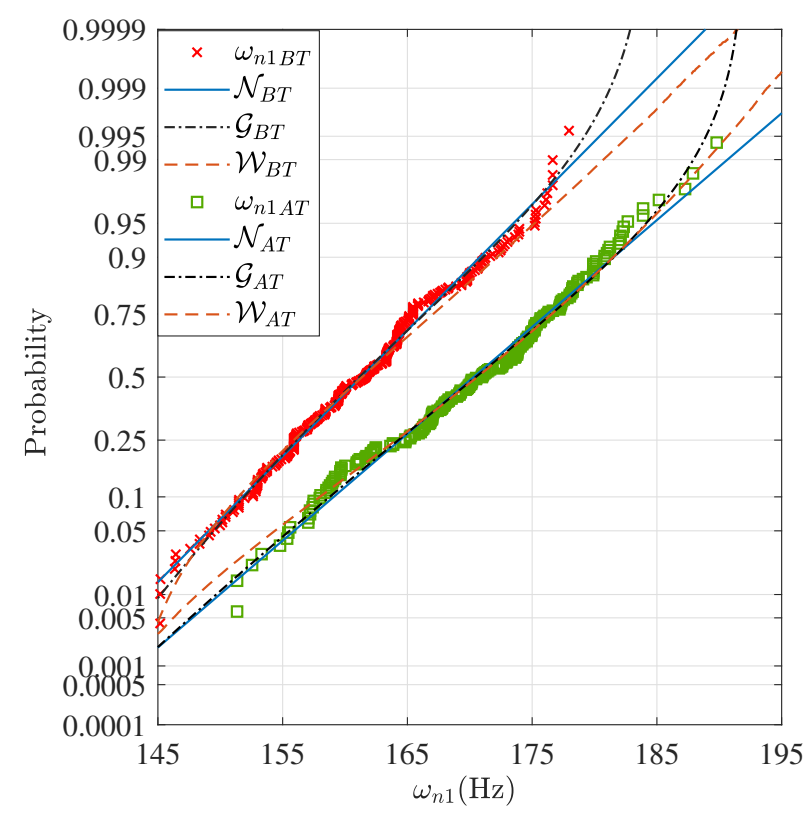

(b) Probability plot.

Figure 5. Histogram and probability plot of the estimated first track resonance frequency before and after the tamping event at location A2 in the switch panel.

defined in the set $\{x \in \mathbb{R}: 1+\xi(x-\mu) / \sigma>0\}$, where $\mu$, $\sigma$ and $\xi \neq 0$ are the location, scale and shape parameters, respectively. The maximum likelihood estimates (MLEs) of these parameters are shown in Table 2.

\section{$\mathcal{G}$-GLR detector design}

In order to detect the degradation of the ballast layer, a generalized likelihood ratio test (GLRT) algorithm is employed using the $\mathcal{G}$ distribution. The following hypothesis testing is set up for monitoring changes in the ballast layer over time

$$
\begin{aligned}
& \mathcal{H}_{0}: \boldsymbol{\theta}=\boldsymbol{\theta}_{0} \\
& \mathcal{H}_{1}: \boldsymbol{\theta} \neq \boldsymbol{\theta}_{0}
\end{aligned}
$$

where $\boldsymbol{\theta}_{0}=\left[\mu_{0}, \sigma_{0}, \xi_{0}\right]^{\mathrm{T}}$ is the vector of parameters of the $\mathcal{G}$ distribution fitted to the data corresponding to the one-month period after the tamping event $\left(\boldsymbol{\omega}_{n 1 A T}\right)$, and $\hat{\boldsymbol{\theta}}$ is the current estimate of the parameter vector based on the distribution fitted to the data collected during monthly intervals.

The $\mathcal{G}$-GLR detector rejects the $\mathcal{H}_{0}$ condition if

$$
L_{G}\left(\boldsymbol{\omega}_{n, 1}\right)=\frac{\mathcal{G}\left(\boldsymbol{\omega}_{n, 1} ; \hat{\boldsymbol{\theta}}, \mathcal{H}_{1}\right)}{\mathcal{G}\left(\boldsymbol{\omega}_{n, 1} ; \boldsymbol{\theta}_{\mathbf{0}}, \mathcal{H}_{0}\right)}>\gamma
$$

where $\gamma$ is the threshold calculated to obtain a desired probability of false alarms. The distribution of $N$ independent and identically distributed (iid) samples of the estimated first track resonance frequencies $\left(\boldsymbol{\omega}_{n, 1}\right)$ is a realization of a $\mathcal{G}$ random process. By taking the natural logarithm of both sides of Eq. (3) the test statistic for the $\mathcal{G}$-GLR can be obtained as follow

$$
\begin{aligned}
g= & N \ln \left(\frac{\sigma_{0}}{\hat{\sigma}}\right)-\left(1+\frac{1}{\hat{\xi}}\right) \sum_{i=1}^{N} \ln \left[1+\hat{\xi}\left(\frac{\omega_{n 1, i}-\hat{\mu}}{\hat{\sigma}}\right)\right] \\
& +\left(1+\frac{1}{\xi_{0}}\right) \sum_{i=1}^{N} \ln \left[1+\xi_{0}\left(\frac{\omega_{n 1, i}-\mu_{0}}{\sigma_{0}}\right)\right] \\
& -\sum_{i=1}^{N}\left[1+\hat{\xi}\left(\frac{\omega_{n 1, i}-\hat{\mu}}{\hat{\sigma}}\right)\right]^{-\frac{1}{\hat{\xi}}} \\
& +\sum_{i=1}^{N}\left[1+\xi_{0}\left(\frac{\omega_{n 1, i}-\mu_{0}}{\sigma_{0}}\right)\right]^{-\frac{1}{\xi_{0}}}
\end{aligned}
$$

where $\hat{\mu}, \hat{\sigma}$, and $\hat{\xi}$ are MLEs of the parameters of $\mathcal{G}$ distribution.

Choosing a proper detection window size for calculating the test statistics is a trade-off between the accuracy and resolution of the degradation index. In order to provide monthly resolution with sufficient accuracy clearly indicating the temporal degradation trend, a 3 month sliding window with 2 month overlap is considered to calculate the test statistic at the end of each month for the period September 2017 - September 2018. It is worth noting that due to the maintenance of the track-side measurement system data are missing in the period January 2017 - September 2017. Figures 6 show the results (i.e., test statistics) of the $\mathcal{G}$-GLR change detection analysis for locations A2, A7 and A11 at Tommerup station. The detector is capable of monitoring the gradual deterioration process in the ballast layer. Noteworthy that since the scale parameter $\sigma$ varies from one month to the other, the data batch size used by the GLR algorithm has a significant influence on the test statistics given in Eq. (4). For a given location along the turnout, the number of estimated frequencies may be different for each monthly period. Moreover, for a given period the number of frequency estimations for different locations is not the same. These are due to the fact that the number of estimations depends on the number of train passage in each monthly period, the number of recorded good-quality signals, and the number of wheel loads properly exciting the substructure. To compare the test statistics calculated for different locations along the turnout, the same number of estimated frequencies are chosen for all locations in each monthly period. 
Table 2. Estimated parameter values of the selected distributions for data collected before and after the tamping event.

\begin{tabular}{ccccc}
\hline Location & Ballast resonance & Distribution & Parameters & $p$-value \\
\hline \multirow{3}{*}{$A_{2}$} & \multirow{2}{*}{$\omega_{n 1, B T}$} & $\mathcal{G}$ & $\xi=-0.18, \sigma=6.59, \mu=158.85$ & 0.05 \\
& & $\mathcal{W}$ & $\alpha=2.89, \beta=21.10, \mu=142.78$ & 0.02 \\
& $\mathcal{N}$ & $\sigma=6.99, \mu=161.62$ & 0.02 \\
\hline \multirow{3}{*}{$A_{2}$} & $\omega_{n 1, A T}$ & $\mathcal{G}$ & $\xi=-0.31, \sigma=8.36, \mu=167.41$ & 0.44 \\
& & $\mathcal{W}$ & $\alpha=4.81, \beta=37.09, \mu=136.23$ & 0.43 \\
& & $\mathcal{N}$ & $\sigma=8.12, \mu=170.21$ & 0.02 \\
\hline
\end{tabular}

\section{Operational ballast quality indicator}

In order for the $\mathcal{G}$-GLR test statistics $g$ to be an interpretable indicator to support the maintenance decision making process, it is paramount to relate its output to a fixed measure of quality of the ballast. For this purpose it is sought to identify an empirical relationship between the test statistics $g$ and longitudinal track quality $\sigma_{H}$. The test statistics is an incremental index whose variation is associated with the change in the estimated ballast resonance frequency, which is in turn related to the rate of degradation of the ballast stiffness. The track stiffness, heavily impacted by the ballast stiffness, directly affects the magnitude of the dynamic loads responsible for the track geometry deterioration (28), which is captured by the variation in longitudinal track quality $\Delta \sigma_{H}$. Hence a mapping between the test statistics $g$ and $\Delta \sigma_{H}$ is determined. To achieve this, the measured data from the track recording car after the tamping event is exploited, and for each location along the turnout the variation of $\sigma_{H}$ with respect to its value measured right after tamping $\sigma_{H, A T}$ is obtained as,

$$
\Delta \sigma_{H}(m)=\sigma_{H}(m)-\sigma_{H, A T}, \quad m=3, \ldots, 8
$$

where $m$ denotes the measurement campaign. Note that $m=$ 1 refers to the geometry campaign before the tamping event and $m=2$ is the campaign right after tamping.

In (29) a monotonic decreasing power function was empirically determined based on measurements of longitudinal level and track stiffness; however due to the limited amount of available track geometry campaigns (six in total in the considered period) it is not possible to confirm such power law. Instead a linear model is found to well describe the relationship between the variation of standard deviation of the track longitudinal level $\Delta \sigma_{H}$ and the first track resonance frequency, which is the adopted indicator of ballast quality in the proposed method (see the right plot in Fig. 7), that is

$$
\Delta \sigma_{H}=f_{i}\left(\omega_{n 1}\right)=\alpha_{i} \omega_{n 1}+\beta_{i}, \quad \alpha_{i}<0, \beta_{i}>0
$$

and $i$ refers to the turnout locations $\mathrm{A} 2, \mathrm{~A} 7$ or A11.

The analytical model of the test statistics $g$ in Eq. (4) shows that when the difference $\left(\omega_{n 1}-\mu\right)$ is small then the logarithm terms dominates over the power terms (in the studied cases $-1<\xi<0$, hence the power terms in Eq.(4) are higher than quadratic, see Table 2). This suggests that in a neighborhood of the location parameter $\mu$ the functional relationship between $g$ and $\omega_{n 1}$ may be linear or sublinear. The empirical analysis for all three considered track sections (A2, A7 and A11) shows that a linear model well describes this mapping, as illustrated in the left plot of Fig. 7. Note that $g$ has been normalized with respect to its maximum value before the tamping event $\left(\bar{g}=g / \max \left(g_{B T}\right)\right)$ to provide a similar range of variation to $\Delta \sigma_{H}$. Hence

$$
\bar{g}=h_{i}\left(\omega_{n 1}\right)=\delta_{i} \omega_{n 1}+\gamma_{i}, \quad \delta_{i}<0, \gamma_{i}>0
$$

and $i$ refers to the turnout location $\mathrm{A} 2, \mathrm{~A} 7$ or $\mathrm{A} 11$.

Since both mappings, $f_{i}\left(\omega_{n 1}\right)$ and $h_{i}\left(\omega_{n 1}\right)$, are linear, then for a given track section $i$ the functional relationship between $\Delta \sigma_{H}$ and $\bar{g}$ is linear, that is

$$
\Delta \sigma_{H}=g_{i}\left(h_{i}^{-1}(\bar{g})\right)=\frac{\alpha_{i}}{\delta_{i}} \bar{g}+\beta_{i}-\frac{\alpha_{i} \gamma_{i}}{\delta_{i}} .
$$

To obtain a single mapping between the $\mathcal{G}$-GLR test statistics and the longitudinal track quality valid across the whole turnout, the functional relationship $\bar{g}-\Delta \sigma_{H}$ is determined empirically. Figure 8 shows that a linear regression model suffices to describe such relation, also when all turnout sections are considered together. Hence an estimator of the variation of longitudinal track quality is given by

$$
\widehat{\Delta \sigma_{H}}=\varepsilon \bar{g}+\zeta, \quad \varepsilon>0, \zeta>0 .
$$

By knowing the value of $\sigma_{H}$ right after a tamping event $\left(\sigma_{H, 0}\right)$, the regression model Eq. (9) and the value of the test statistics $g$ can be used to estimate the standard deviation of the longitudinal level $\hat{\sigma}_{H}$ at any given point in time in between track geometry measurement campaigns

$$
\hat{\sigma}_{H}(k)=\sigma_{H, 0}+\widehat{\Delta \sigma_{H}}(\bar{g}(k)),
$$

where $k$ is the time index at which the test statistics $g$ is computed. This provide an operational indicator of ballast quality since $\hat{\sigma}_{H}$ can be assessed against the track quality classes defined in the European standard; therefore its value can be utilized to schedule ballast tamping actions.

Figure 9 shows the estimated values of $\sigma_{H}$ obtained for locations A2, A7 and A11. The gray box shows the period in which the data is missing due to the maintenance of the measurement system. The proposed estimator $\hat{\sigma}_{H}$ provides reliable estimates in good agreement with the values of $\sigma_{H}$ computed based on the measured longitudinal track variation. The quality of ballast can then be determined over time by using the values of $\hat{\sigma}_{H}$ and considering the classification in Table 1 provided by the European standard. This information may be employed by the track infrastructure manager to plan the ballast maintenance activities.

\section{Concluding remarks}

The integration of novel intelligent monitoring systems into the legacy systems used by railway infrastructure managers 


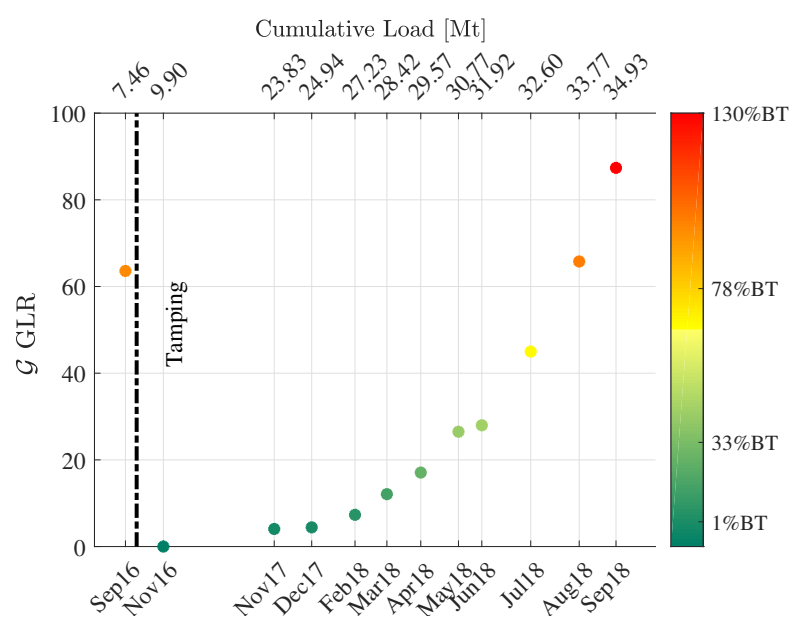

(a) A2 location

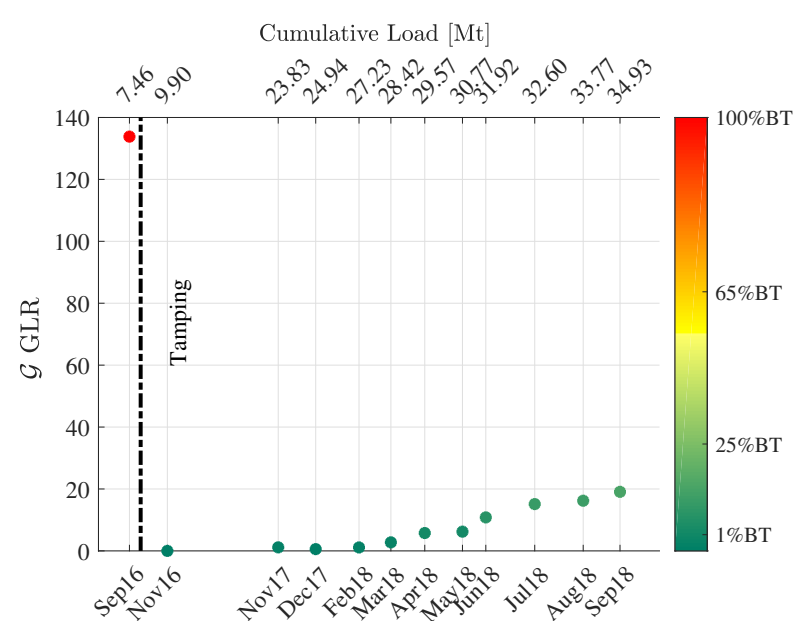

(b) A7 location

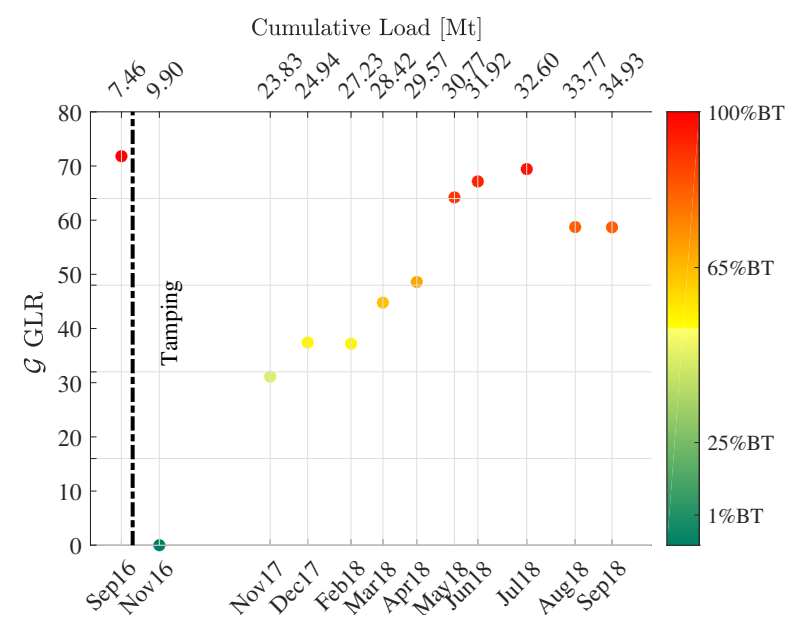

(c) A11 location

Figure 6. $\mathcal{G}$-GLR for different locations along the turnout

requires the ability of the former to output interpretable and actionable information that can be exploited for maintenance decision making. The paper addressed the process of making operational the ballast degradation monitoring system proposed in (1) by converting its output into an estimate of $\sigma_{H}$, which is the parameter already used by
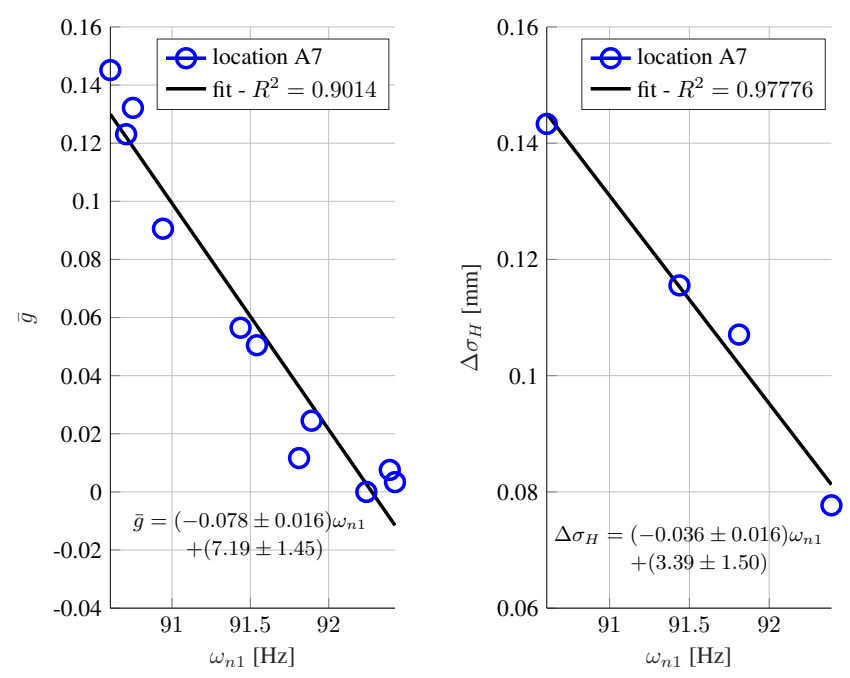

Figure 7. Linear regression models of $\bar{g}$ and $\Delta \sigma_{H}$ as function of the first track resonance frequency $\omega_{n 1}$.

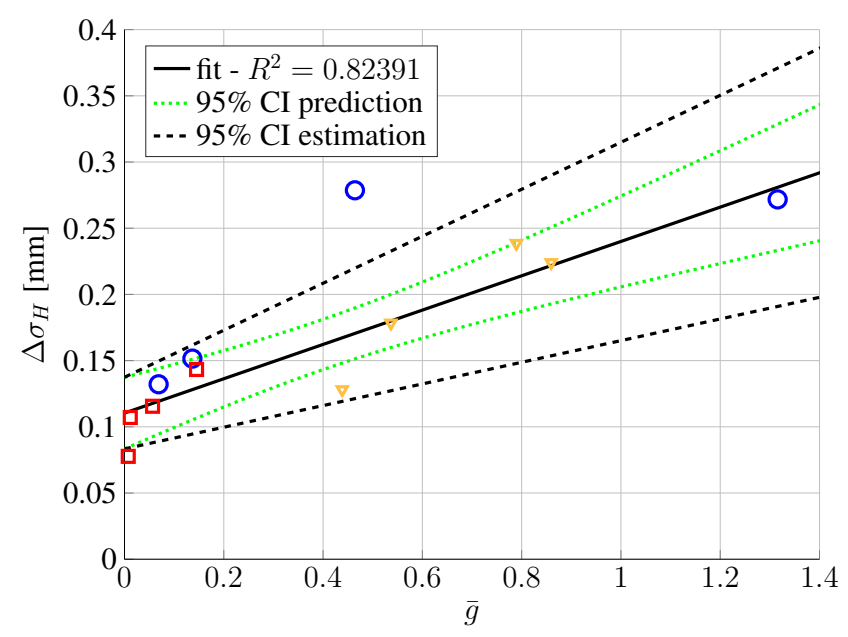

Figure 8. Linear fit to model $\Delta \sigma_{H}$ with respect to $\bar{g}$. Legend: A2 - blue dots; A7 - orange dots; A11 - yellow dots.

\section{Cumulative Load [Mt]}

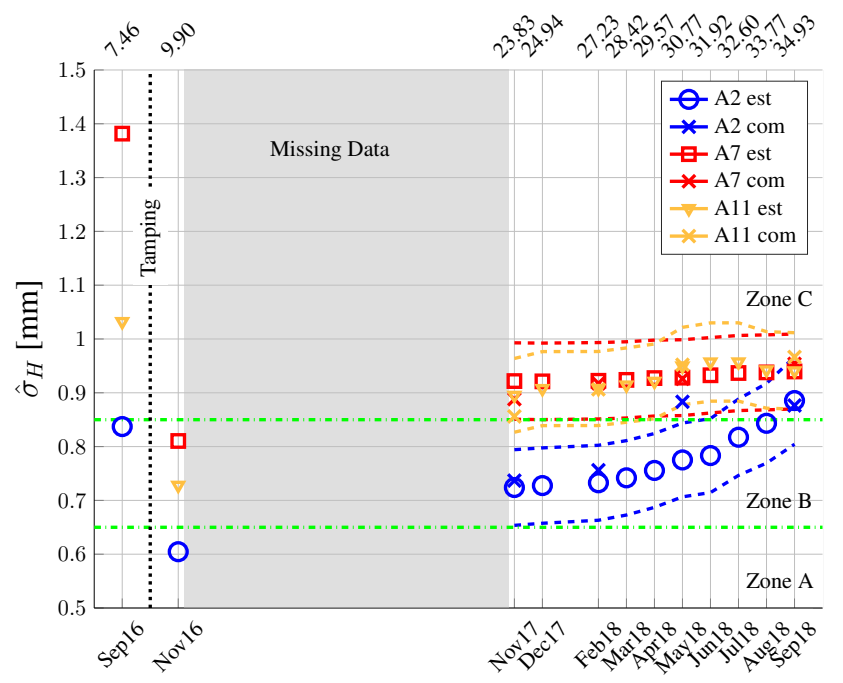

Figure 9. Estimated $\sigma_{H}$ for locations $\mathrm{A} 2, \mathrm{~A} 7$ and $\mathrm{A} 11$ with the respective $95 \%$ confidence interval. The track quality classes as defined in the European standard are marked in the plot. 
infrastructure managers to evaluate the longitudinal track quality and call for ballast tamping.

Exploiting track geometry data as well as track vibration data for a single turnout over a period of two years, a linear regression model was proposed to map the output of a statistical change detection algorithm based on the GEV distribution into the variation of longitudinal track geometry. The test statistics for ballast monitoring was shown to remarkably identify different degradation trends at distinct locations along the turnout; however its numerical output provided only a relative measure of ballast quality in comparison to how the ballast was at the time of last tamping. By combining the test statistics with the regression model the resulting ballast monitoring system relates changes in ballast quality (stiffness) as seen from variations in the first track resonance frequency to changes in longitudinal track geometry.

The obtained estimate $\hat{\sigma}_{H}$ was shown to well match the value of $\sigma_{H}$ directly computed from the longitudinal track level measured during four consecutive track geometry campaigns after a tamping event. Moreover, since the test statistics can be computed with an arbitrary time resolution then the ballast monitoring system can predict changes in track longitudinal level in between geometry measurement campaigns.

The proposed system for monitoring of ballast degradation in turnouts potentially outperforms methods currently employed in the industry since it achieves both higher spatial and time resolution of the delivered information while categorizing its output according to the European standards adopted by the infrastructure managers. However, its application entails (i) the introduction of additional measurement systems to acquire vibration data at few selected locations along the turnout; (ii) the verification that the identified linear regression model is descriptive of other turnouts subject to different environmental and operational conditions; (iii) the further assessment that the computation of $\sigma_{H}$ over track sections of $10 \mathrm{~m}$ has sufficient accuracy in describing the local track quality.

\section{Acknowledgements}

This research study has been carried out, as part of the INTELLISWITCH project. The research is financially supported by Innovation Fund Denmark with grant number 4109-00003B. The authors gratefully acknowledge this support.

\section{References}

[1] Barkhordari P, Galeazzi R and Blanke M. Monitoring of turnout ballast degradation using statistical low-complexity behavioural models. Submitted to IEEE Transactions on Control Systems Technology 2019; .

[2] Juul Andersen K. Årsrapport 2012. Technical Report 1300144, banedanmark, 2012.

[3] Hassankiadeh S. Failure Analysis of Railway Switches and Crossings for the purpose of Preventive Maintenance. MSc Thesis, Royal Institute of Technology, 2011.

[4] Lam H, Wong M and Yang Y. A feasibility study on railway ballast damage detection utilizing measured vibration of in situ concrete sleeper. Engineering Structures 2012; 45: 284 298.

[5] Barkhordari P, Galeazzi R, de Miguel Tejada A et al. Lowcomplexity behavioral model for predictive maintenance of railway turnouts. In Proceedings of Annual Conference of the Prognostics and Health Management Society (2017).

[6] Barkhordari P and Galeazzi R. Statistical model of railway's turnout based on train induced vibrations. In Proceedings of the 10th IFAC Symposium on Fault Detection, Supervision and Safety of Technical Processes (2018).

[7] Berggren E. Railway Track Stiffness - Dynamic Measurements and Evaluation for Efficient Maintenance. PhD Thesis, Royal Institute of Technology, 2009.

[8] Thompson R and Li D. Automated vertical track strength testing using ttcis track loading vehicle. Technology Digest February 2002;

[9] Norman C, Farritor S, Arnold R et al. Design of a system to measure track modulus from a moving railcar. US Department of Transportation, Federal Railroad Administration, Office of , 2006.

[10] Berggren EG, Kaynia AM and Dehlbom B. Identification of substructure properties of railway tracks by dynamic stiffness measurements and simulations. Journal of Sound and Vibration 2010; 329(19): 3999-4016.

[11] Le Pen L, Milne D, Thompson D et al. Evaluating railway track support stiffness from trackside measurements in the absence of wheel load data. Canadian Geotechnical Journal 2016; 53(7): 1156-1166.

[12] Nissen A. Innovative intelligent rail, embedded \& integrated sensors: Systems design hierarchy report. Technical Report H2020-MG-2014, Deliverable D2.3, In2Rail, 2017.

[13] CEN. En 13848-6, railway applications-track-track geometry quality-part 6: characterisation of track geometry quality, 2014.

[14] Mishra M, Odelius J, Thaduri A et al. Particle filter-based prognostic approach for railway track geometry. Mechanical Systems and Signal Processing 2017; 96: 226-238.

[15] Nielsen JC and Li X. Railway track geometry degradation due to differential settlement of ballast/subgrade-numerical prediction by an iterative procedure. Journal of Sound and Vibration 2018; 412: 441-456.

[16] Gatin O, LHenoret B, Isasi A et al. Track geometry condition monitoring system for non intrusive measurements on commercial trains based on wireless sensor networks. In 10th World Congress Railway Research (WCRR) (2013).

[17] Quiroga LM and Schnieder E. Monte carlo simulation of railway track geometry deterioration and restoration. Proceedings of the Institution of Mechanical Engineers, Part O: Journal of Risk and Reliability 2012; 226(3): 274-282.

[18] Weston P, Roberts C, Yeo G et al. Perspectives on railway track geometry condition monitoring from in-service railway vehicles. Vehicle System Dynamics 2015; 53(7): 1063-1091.

[19] Nissen A. Development of life cycle cost model and analyses for railway switches and crossings. $\mathrm{PhD}$ Thesis, Luleå tekniska universitet, 2009.

[20] Nissen A, Parida A and Kumar U. Condition monitoring of railway switches and crossing by using data from track recording cars. International Journal of COMADEM 2010; 13(3): 36-40. 
[21] Hovad E, Andersen JF, Thyregod C et al. Spatial alignment of track recording car data in turnouts. In Fourth International Conference on Railway Technology: Research, Development and Maintenance. Sitges, Barcelona, Spain.

[22] Khouy IA, Larsson-Kråik PO, Nissen A et al. Geometrical degradation of railway turnouts: a case study from a swedish heavy haul railroad. Proceedings of the Institution of Mechanical Engineers, Part F: Journal of Rail and Rapid Transit 2014; 228(6): 611-619.

[23] Minbashi N, Bagheri M, Golroo A et al. Use of power spectral density for effective turnout geometry maintenance. Transportation Research Record 2016; 2545(1): 79-88.

[24] Asadzadeh SM, Galeazzi R, Hovad E et al. Ballast degradation modeling for turnouts based on track recording car data. In PHM Society European Conference (2018), volume 4 .
[25] Barkhordari P, Galeazzi R, de Miguel Tejada A et al. Behavioural models for railway turnouts monitoring predictiveness, robustness, portability and scalability. Submitted to Mechanical Systems and Signal Processing 2018; .

[26] Soleimanmeigouni I, Ahmadi A and Kumar U. Track geometry degradation and maintenance modelling: A review. Proceedings of the Institution of Mechanical Engineers, Part F: Journal of Rail and Rapid Transit 2018; 232(1): 73-102.

[27] Asadzadeh SM and Galeazzi R. The predictive power of track dynamic response for monitoring of ballast degradation in turnouts. Submitted to Journal of Rail and Rapid Transit 2018; .

[28] Lundqvist A and Dahlberg T. Dynamic train/track interaction including model for track settlement evolvement. Vehicle system dynamics 2004; 41: 667-676.

[29] Puzavac L, Popović Z and Lazarević L. Influence of track stiffness on track behaviour under vertical load. PROMETTraffic \& Transportation 2012; 24(5): 405-412. 